\title{
Deconvolution Process of High-Resolution HAADF STEM Images
}

\author{
M. Shiojiri*, K. Watanabe , N. Nakanishi $^{* * *}$, T. Yamazaki*** ${ }^{* *}$ M. Kawasaki****, \\ A. Recnik ${ }^{* * * * *}$ and M. Ceh ${ }^{* * * * *}$ \\ *Kyoto Institute of Technology, 606-8585, Japan \\ *** Tokyo Metropolitan College of Technology, 140-0011, Japan \\ *** Department of Phys ics, Tokyo University of Science, 162-8601, Japan \\ ***** JEOL USA Inc. Dearborn Road, MA. 01960 \\ ****** Institute of Jož ef Stefan, 1000 Ljubljana, Slovenia
}

High-resolution high-angle annular dark field (HAADF) scanning transmission electron microscopy (STEM) has been widely used to structural analysis of materials. HAADF STEM images do not show contrast reversal against thickness and defocus unlike conventional HRTEM images, and an HAADF STEM image is given by a convolution between the probe function (the intensity of a convergent probe) and the object function in which each atomic column acts as an independent scattering center. Experimental images that are at poor resolution or have artifacts as results of the convolution can be transferred to the object function by a deconvolution process, as seen in Fig. 1. This paper describes our new scheme for high-resolution HAADF STEM image process, where the deconvolution is combined with maximum entropy method [1]. This has been extended to process of an image distorted systematically during STEM recording. The image distortion is frequently caused by instrumental instability in field-emission electron microscopes of today.

Figs 2a and 2c show two experimental [110] Si images recorded using a probe at a semiangle of 17 mrad and a 35 90 mrad detector in a JEM 2010F-TEM/STEM $(C \mathrm{~s}=1.0 \mathrm{~mm})$ operated at $200 \mathrm{keV}$. The most probable defoci were determined to be $\Delta f=-63$ and $-75 \mathrm{~nm}$ from the maxima of entropies calculated for these images, respectively. Figs $2 b$ and $2 d$ show deconvoluted images with the probes assigned by these defoci. The deconvolution retrieves the experimental images to object functions approximated to the projected atomic structure image. Practically, a threshold value of the probe function $p_{\text {thre }}$ is set up to exclude intensity for $\left|p^{\text {eff }}(\boldsymbol{G})\right| \leq p_{\text {thre }}$, where $p^{\text {eff }}(\boldsymbol{G})$ is Fourier transform of the probe function and $p_{\text {thre }}$ is a level below which the intensity is subtracted. Hence, mise can also be eliminated in this process.

We have processed high-resolution HAADF STEM images where the lattice fringes were systematically elongated and/or sheared during recording due to instrumental and technical problem. The process includes, as a pretreatment of the deconvolution, a correction of the diffractogram of HAADF STEM image referring the diffractogram of a HRTEM image (or electron diffraction pattern) of the same area. An example is shown in Fig. 3. A HAADF image of [01 10] ZnO (Fig. 3a), which was recorded using a 20-mrad-semiangle probe with a lens of $C \mathrm{~s}=0.5 \mathrm{~mm}$ and a 100 200-mrad detector, was retrieved as shown in Fig. 3f. The lattice of the image was completely reformed, and a processed image, deconvoluted using a probe at $\Delta f=-37 \mathrm{~nm}$, can be regarded as the object function or the projected atomic structure. Thus, we can get a real $Z$-contrast structure image, retrieved by removing the instrumental deformation and the influence of the beam probe.

Reference

[1] K. Watanabe et al., Ultramicroscopy (2002) in press. 

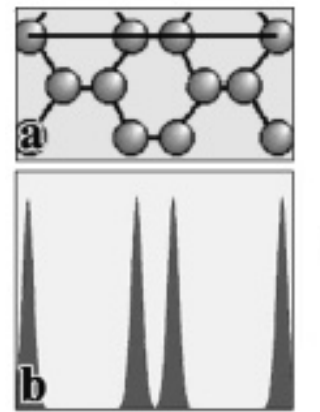

Object (Structure)

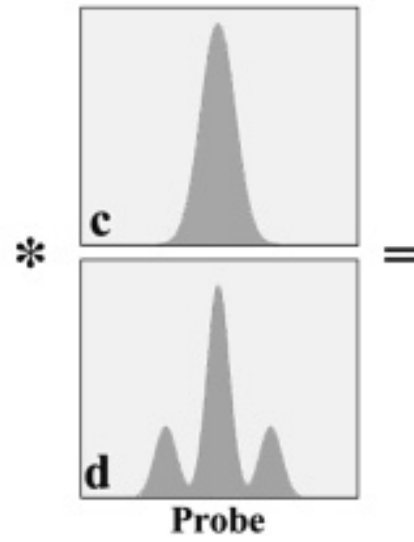

Probe

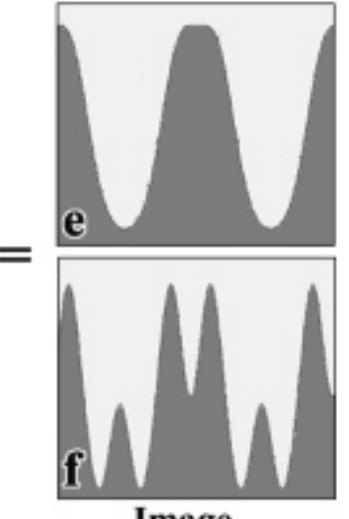

Image

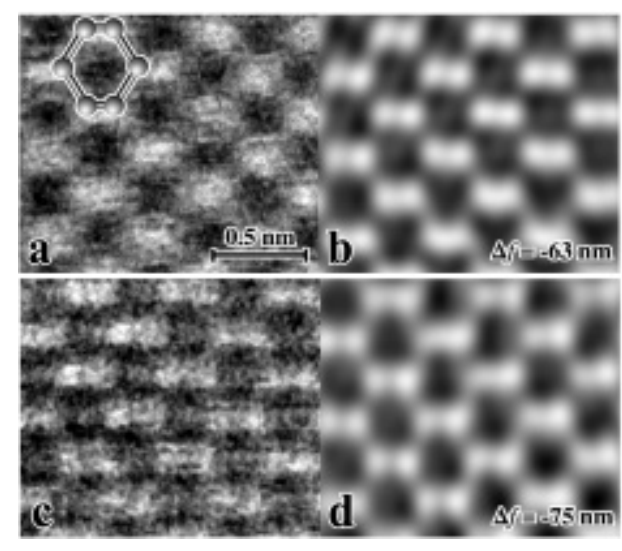

FIG. 2 a and c Experimental HAADF STEM images of [011] Si $85 \mathrm{~nm}$ thick, recorded at different defoci. b and d Deconvoluted images of (a) and (c), respectively, using $p_{\text {thre }}=0.01$. the a semiangle of the probes $\alpha=17$ mrad), respectively. e and $\mathbf{f}$ Observed images deformed with the probes (c) and (d), respectively.
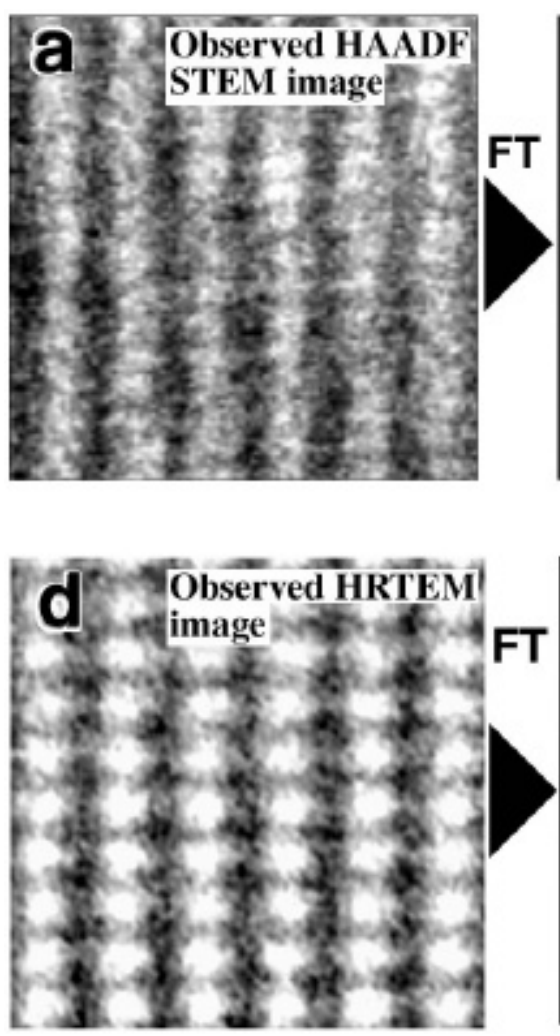

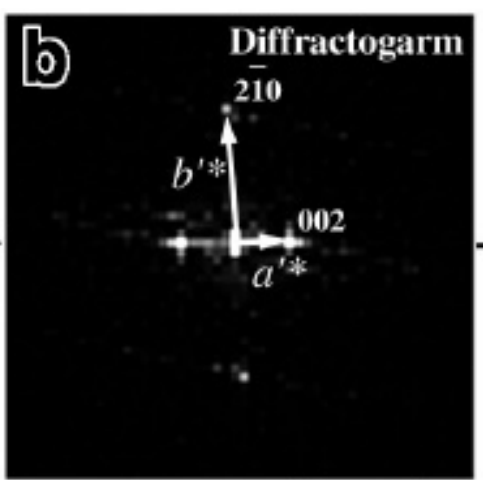

Correct spot position
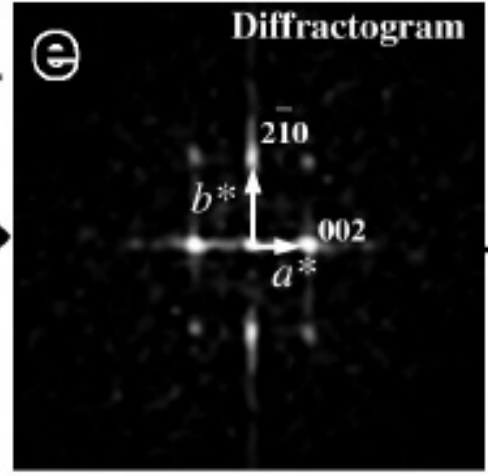
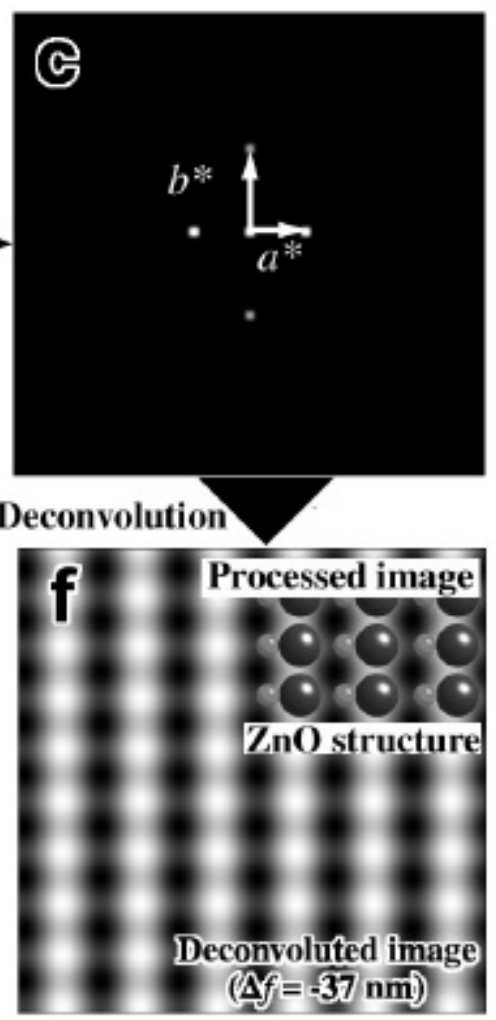

FIG. 3 Image process of an experimental HAADF STEM [0110] ZnO image a, which was deformed by convolution with the probe and also the instrumental instability during STEM recording. The deconvolution process was made using diffractogram $\mathbf{c}$ and $p^{\text {eff }}(\boldsymbol{G})$ at $\Delta f=-37$ $\mathrm{nm}$. Retrieved image $\mathbf{f}$ by this process shows a real $Z$-contrast structure image. 